\title{
Resonance-enhanced group delay times in an asymmetric single quantum barrier
}

\author{
Chun-Fang $\mathrm{Li}^{1}$ \\ Department of Physics, Shanghai University, 99 Shangda Road, Shanghai 200444, People's \\ Republic of China \\ State Key Laboratory of Transient Optics Technology, Xi'an Institute of Optics and Precision \\ Mechanics, Academia Sinica, 322 West Youyi Road, Xi'an 710068, People's Republic of China
}

\begin{abstract}
It is shown that the transmission and reflection group delay times in an asymmetric single quantum barrier are greatly enhanced by the transmission resonance when the energy of incident particles is larger than the height of the barrier. The resonant transmission group delay is of the order of the quasibound state lifetime in the barrier region. The reflection group delay can be either positive or negative, depending on the relative height of the potential energies on the two sides of the barrier. Its magnitude is much larger than the quasibound state lifetime. These predictions have been observed in a microwave experiment by $\mathrm{H}$. Spieker of Braunschweig University.
\end{abstract}

PACS numbers: 03.65.Xp, 73.23.Ad

\footnotetext{
${ }^{1}$ Mailing address: Department of Physics, Shanghai University, 99 Shangda Road, Shanghai 200444, People's Republic of China. Email: cfli@staff.shu.edu.cn
} 
The tunnelling time of particles through single or multiple quantum barriers has drawn much attention [1, 2, 3, 4, 5] with the advent of techniques for the fabrication of semiconductor tunnelling devices, such as single-electron tunnelling transistors [6], resonant tunnelling diodes [7, quantum cascade lasers [8, and resonant photodetectors [9]. Theoretical [10, 11, 12, 13, 14, 15, 16, 17, 18, 19] as well as experimental 2, 20, 21, 22, 23, 24, 25, 26, 27, investigations have been extensively made on this problem. It was found that the group delay (also referred to as the phase time in the literature [1]) for particles tunnelling through a potential barrier saturates to a constant value in the opaque limit [4, 28, 29, 30. This is the so-called Hartman effect [31. The reflection group delay is the same as the transmission one in a symmetric configuration [15. It will be shown in this Letter that the reflection group delay from a single barrier of asymmetric configuration can be negative and is greatly enhanced by the transmission resonance.

The negative group delays in both reflection and transmission were previously discovered. But they all occur in quantum-well structures, such as single quantum wells 32, 33, 34, double-barrier quantum wells 35, 36, and their optical analogues [5, 37. What we consider here is such a case in which particles are scattered by an asymmetric single barrier, the height of which is less than the energy of incident particles. Quasibound states were predicted and observed in such a situation 38 . This is a classically allowed motion. The particle in the barrier region has a real classical moving velocity which specifies a classical traversal time $\tau_{c}$. It is found that the transmission and reflection group delays are both enhanced by transmission resonance. The reflection group delay can be either positive or negative, depending on the relative height of the potential energies on the two sides of the barrier. The negative resonant reflection group delay corresponds to a transmission probability that is larger than 1 . The resonant transmission group delay is of the order of the quasibound state lifetime in the barrier region and is larger than the classical time $\tau_{c}$. And the magnitude of resonant reflection group delay is much larger than the lifetime of the quasibound state in the barrier region. The reflected wave packet is considered without taking into account the interference between the incident and the reflected waves [1, 35].

The height of the potential barrier, extending from 0 to $a$, is $V_{0}$. The values of the potential energies on the left and right handed sides of the barrier are $V_{1}$ and $V_{2}$, respectively. It is assumed that $V_{0}>V_{1}$ and $V_{0}>V_{2}$. Let a beam of particles be incident from the left, and let be $\psi_{i n}(x)=$ $A \exp \left(i k_{1} x\right)$ the Fourier component of the incident wave packet, where $k_{1}=\left[2 \mu\left(E-V_{1}\right)\right]^{1 / 2} / \hbar$, and $\mu$ is the mass of incident particles. In the following, we will assume that the energy, $E$, of incident particles is larger than the height, $V_{0}$, of the potential barrier. Denoting, respectively, by $B \exp \left(-i k_{1} x\right)$ and $F \exp \left[i k_{2}(x-a)\right]$ the corresponding Fourier components of the reflected and transmitted wave packets, then the Schrödinger equation and boundary conditions at $x=0$ and $x=a$ give $r \equiv \frac{B}{A}=\frac{g_{1}}{g_{2}} \exp \left[i\left(\phi_{2}-\phi_{1}\right)\right]$, and $t \equiv \frac{F}{A}=\frac{1}{g_{2}} \exp \left(i \phi_{2}\right)$, where $k_{2}=\left[2 \mu\left(E-V_{2}\right)\right]^{1 / 2} / \hbar$, 
$k_{0}=\left[2 \mu\left(E-V_{0}\right)\right]^{1 / 2} / \hbar$, non-negative number $g_{1}$ and real number $\phi_{1}$ are defined by a complex number as follows,

$$
g_{1} \exp \left(-i \phi_{1}\right)=\frac{1}{2}\left(1-\frac{k_{2}}{k_{1}}\right) \cos \left(k_{0} a\right)-\frac{i}{2}\left(\frac{k_{2}}{k_{0}}-\frac{k_{0}}{k_{1}}\right) \sin \left(k_{0} a\right),
$$

and non-negative number $g_{2}$ and real number $\phi_{2}$ are defined similarly by another complex number as follows,

$$
g_{2} \exp \left(-i \phi_{2}\right)=\frac{1}{2}\left(1+\frac{k_{2}}{k_{1}}\right) \cos \left(k_{0} a\right)-\frac{i}{2}\left(\frac{k_{2}}{k_{0}}+\frac{k_{0}}{k_{1}}\right) \sin \left(k_{0} a\right) .
$$

According to definition (1), we have

$$
\tan \phi_{1}=\frac{1 / k_{0}-k_{0} / k_{1} k_{2}}{1 / k_{2}-1 / k_{1}} \tan \left(k_{0} a\right)
$$

which shows that $\phi_{1}$ will change its sign by exchanging $k_{1}$ and $k_{2}$. This property will have important effect on the group delay of reflected particles. According to Eq. (2), we have

$$
\tan \phi_{2}=\frac{1 / k_{0}+k_{0} / k_{1} k_{2}}{1 / k_{2}+1 / k_{1}} \tan \left(k_{0} a\right)
$$

which shows that $\phi_{2}$ is symmetric between $k_{1}$ and $k_{2}$. We can also see from Eqs. (3) and (4) that $\phi_{1}$ and $\phi_{2}$ can be exchanged from one to another by changing the sign of $k_{1}$. This symmetry between $\phi_{1}$ and $\phi_{2}$ will simplify our calculation of the group delay.

First, let us look at the group delay $\tau_{t}$ of transmitted particles. It is defined as $\hbar\left(d \phi_{2} / d E\right)$ [39 [15] and is given by

$$
\tau_{t}=\frac{\tau_{c}}{4 g_{2}^{2}}\left(1+\frac{k_{2}}{k_{1}}\right)\left[\frac{k_{2}}{k_{0}}+\frac{k_{0}}{k_{1}}-\left(1-\frac{k_{0}^{2}}{k_{1}^{2}}\right)\left(\frac{k_{2}}{k_{0}}-\frac{k_{0}}{k_{2}}\right) \frac{\sin 2 k_{0} a}{2 k_{0} a}\right],
$$

where $\tau_{c}=a / v_{c}$ is the time taken for classical particles to travel through the barrier region, $v_{c}=$ $\frac{1}{\hbar\left(d k_{0} / d E\right)}=\frac{\hbar k_{0}}{\mu}$ is the classical velocity of the particles in the barrier region. It is clear that $\tau_{t}$ is in general different from the classical time $\tau_{c}$. Furthermore, it is easy to show that $\tau_{t}$ can be larger as well as less than $\tau_{c}$. In fact, when $k_{0} a=m \pi(m=1,2,3 \ldots), \tau_{t}$ reduces to

$$
\left.\tau_{\text {tmax }} \equiv \tau_{t}\right|_{k_{0} a=m \pi}=\frac{k_{1} k_{2}+k_{0}^{2}}{k_{0}\left(k_{1}+k_{2}\right)} \tau_{c}
$$

If the energy of incident particles is so close to the height of the potential barrier that $k_{0}$ is much less than $k_{1}$ and $k_{2}, \tau_{\text {tmax }}$ will be larger than $\tau_{c}$. On the other hand, when $k_{0} a=(m+1 / 2) \pi, \tau_{t}$ becomes $\left.\tau_{t}\right|_{k_{0} a=(m+1 / 2) \pi}=\frac{1+k_{2} / k_{1}}{k_{2} / k_{0}+k_{0} / k_{1}} \tau_{c}$. It is less than $\tau_{c}$ under the above-mentioned condition. In Fig. 1 is shown the dependence of $\tau_{t}$ upon the thickness $a$ of the barrier, where $V_{0} / E=0.95$, $V_{1} / E=0, V_{2} / E=0.3$, and $a$ is re-scaled to be $k_{0} a$. We see that $\tau_{t}$ is maximum at $k_{0} a=m \pi$.

It will be useful to introduce a quantity $T$, called transmission probability, which is defined as

$$
T \equiv|t|^{2}=\frac{4 k_{0}^{2} k_{1}^{2}}{k_{0}^{2}\left(k_{1}+k_{2}\right)^{2}+\left(k_{1}^{2}-k_{0}^{2}\right)\left(k_{2}^{2}-k_{0}^{2}\right) \sin ^{2} k_{0} a} .
$$




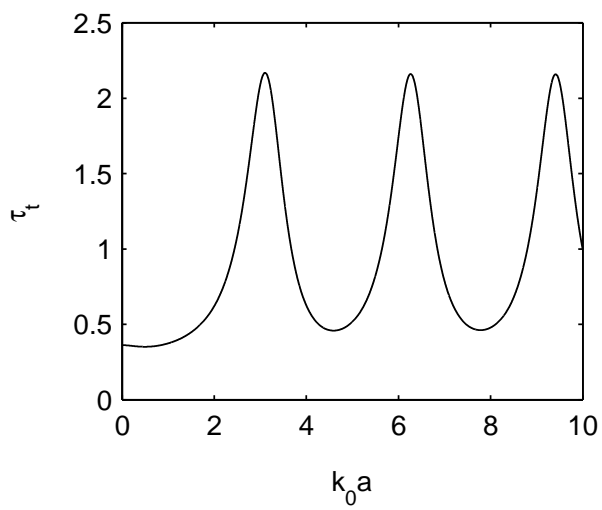

Figure 1: The dependence of the transmission group delay $\tau_{t}$ upon the thickness $a$ of the barrier, where $V_{0} / E=0.95, V_{1} / E=0, V_{2} / E=0.3, a$ is re-scaled to be $k_{0} a$, and $\tau_{t}$ is in units of $\tau_{c}$.

The transmission coefficient, defined as the ratio of the transmitted probability current density to the incident probability current density [40, is then $\left(k_{2} / k_{1}\right) T$. When $k_{0} a=m \pi$, $T$ reaches its maximum, $T_{\max }=\frac{4}{\left(1+k_{2} / k_{1}\right)^{2}}$. The resonance condition $k_{0} a=m \pi$ for transmission through a single barrier is the same as that for the quasilocalization of the states in the barrier region [38. It can be shown that the resonant transmission time (5) is of the order of the quasibound state lifetime in the barrier region [12]. The symmetry of $\phi_{2}$ between $k_{1}$ and $k_{2}$ means that the transmission group delay is the same whether the incident particles come to the barrier from left-handed or right-handed side [15].

Now let us pay our attention to the reflection group delay, which is $\tau_{r}=\tau_{t}+\tau_{1}$ as is seen from the expression of $r$, where

$$
\tau_{1}=-\hbar \frac{d \phi_{1}}{d E}=-\frac{\tau_{c}}{4 g_{1}^{2}}\left(1-\frac{k_{2}}{k_{1}}\right)\left[\frac{k_{2}}{k_{0}}-\frac{k_{0}}{k_{1}}-\left(1-\frac{k_{0}^{2}}{k_{1}^{2}}\right)\left(\frac{k_{2}}{k_{0}}-\frac{k_{0}}{k_{2}}\right) \frac{\sin 2 k_{0} a}{2 k_{0} a}\right],
$$

and

$$
g_{1}^{2}=\frac{1}{4}\left(1-\frac{k_{2}}{k_{1}}\right)^{2} \cos ^{2}\left(k_{0} a\right)+\frac{1}{4}\left(\frac{k_{2}}{k_{0}}-\frac{k_{0}}{k_{1}}\right)^{2} \sin ^{2}\left(k_{0} a\right)
$$

Note:

(1) When the energy of incident particles is so close to the height of the potential barrier that $k_{1} \sim k_{2}$ and $k_{0} \ll k_{2}$, the second term on the right-handed side of Eq. (8) is usually much larger than the first term unless $k_{0} a=m \pi$, at which $g_{1}^{2}$ is minimal. As a result, near its maximum, $\tau_{1}$ can be approximated as

$$
\tau_{1} \approx-\frac{\tau_{c}}{4 g_{1}^{2}}\left(1-\frac{k_{2}}{k_{1}}\right)\left(\frac{k_{2}}{k_{0}}-\frac{k_{0}}{k_{1}}\right)
$$

And its maximum has a value of $\tau_{1 \max }=-\frac{k_{1} k_{2}-k_{0}^{2}}{k_{0}\left(k_{1}-k_{2}\right)} \tau_{c}$. Comparing with Eq. (15), we see that the magnitude of $\tau_{1 \max }$ is much larger than the resonant transmission group delay, which means that 
the reflection group delay, $\tau_{r}$, is dominated by $\tau_{1}$ near resonances, and its sign is determined by the sign of $\tau_{1}$. This shows that the magnitude of the resonant reflection group delay is much larger than the quasibound state lifetime in the barrier region. It is clear from Eq. (9) that $\tau_{1}$ can be negative as well as positive. When $k_{1}>k_{2}\left(V_{1}<V_{2}\right), \tau_{1}$ (and hence $\tau_{r}$ ) is negative. On the other hand, when $k_{1}<k_{2}\left(V_{1}>V_{2}\right), \tau_{1}$ (and hence $\tau_{r}$ ) is positive. These properties of $\tau_{1}$ can also be inferred from Eq. (3). As a result, when the reflection group delay for incidence from the left is positive, it is negative for incidence from the right for the same configuration.

(2) When $k_{1}>k_{2}, T_{\max }>1$. This shows that the negative peaks of the reflection group delay correspond to a transmission probability that is larger than 1 . The fact that the transmission probability can be larger than 1 is not at odds with the law of probability conservation. It is the probability current density, rather than the probability itself, that is in direct connection with probability conservation in quantum scattering. In fact, the transmission coefficient, $\left(k_{2} / k_{1}\right) T$, is always less than 1.

(3) The reflection coefficient $|r|^{2}$ does not vanish at the transmission resonance, so that the reflected wave packet is well defined under the condition that follows (Eq. (10)).

(4) For the case of far from resonances, $k_{0} a=(m+1 / 2) \pi$, $\tau_{1}$ becomes $\left.\tau_{1}\right|_{k_{0} a=(m+1 / 2) \pi}=$ $-\frac{1-k_{2} / k_{1}}{k_{2} / k_{0}-k_{0} / k_{1}} \tau_{c}$. Under the above mentioned conditions $\left(k_{1} \sim k_{2}\right.$ and $\left.k_{0} \ll k_{2}\right)$, its magnitude is much smaller than the corresponding transmission group delay, $\left.\tau_{t}\right|_{k_{0} a=(m+1 / 2) \pi}$. This shows that the reflection group delay is almost the same as the transmission one when the energy of incident particles is far from resonances.

In Fig. 2 is shown the dependence of $\tau_{r}$ upon the thickness $a$ of the barrier, where $a$ is rescaled to be $k_{0} a$. The solid curve corresponds to negative-peak group delay, where all the physical parameters are the same as in Fig. 1. The dashed curve corresponds to positive-peak group delay, where $V_{0} / E=0.95, V_{1} / E=0.3$, and $V_{2} / E=0$. It is seen that the peaks of the group delay occur at $k_{0} a=m \pi$ and are much larger than the peaks of the transmission group delay whether they are negative or positive.

The symmetries of $\phi_{1}$ and $\phi_{2}$ between $k_{1}$ and $k_{2}$ discussed before mean that the transmission group delay and reflection group delay satisfy the average principle [15, $\tau_{r 1}+\tau_{r 2}=2 \tau_{t}$, where $\tau_{r 1}$ and $\tau_{r 2}$ are reflection group delays for the incident particles coming from left-handed side and right-handed side, respectively.

Next, we discuss the validity of the above theoretical results. To this end, let us look at the dependence of the reflection group delay on the energy of incident particles, which is shown in Fig. 3, where $V_{1}=0, V_{2}=0.3 V_{0}, E \in\left[V_{0}, 1.15 V_{0}\right], a=10 /\left(0.3 \mu V_{0}\right)^{1 / 2}$, and the incidence energy $E$ is re-scaled to be $k_{0} a$. The half width of the peak of the reflection group delay, which can be 


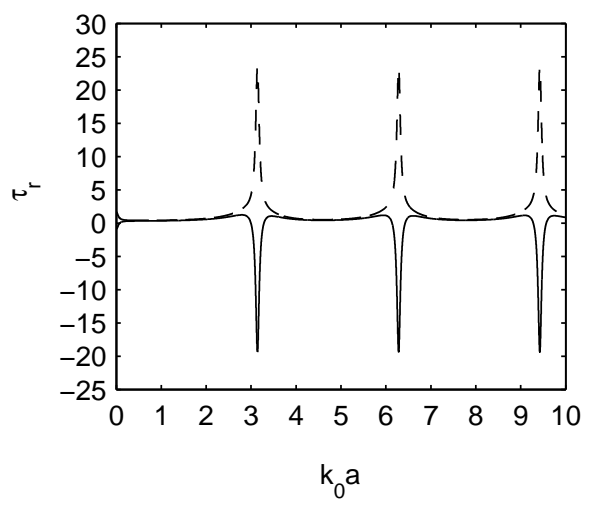

Figure 2: The dependence of the reflection group delay $\tau_{r}$ upon the thickness $a$ of the barrier, where $a$ is re-scaled to be $k_{0} a$, and $\tau_{r}$ is in units of $\tau_{c}$. The solid curve corresponds to the negative-peak group delay, where all the physical parameters are the same as in Fig. 1. The dashed curve corresponds to the positive-peak group delay, where $V_{0} / E=0.95, V_{1} / E=0.3$, and $V_{2} / E=0$.

approximately obtained from its dominant part $\tau_{1}$, is

$$
\Delta E=\frac{\hbar}{\tau_{c}} \sin ^{-1} \frac{k_{0}\left|k_{1}-k_{2}\right|}{\left[\left(k_{1}^{2}-k_{0}^{2}\right)\left(k_{2}^{2}-k_{0}^{2}\right)\right]^{1 / 2}} .
$$

For a Gaussian-shaped wave packet, its energy half-width $\delta E$ is related to its time spreading $w$ by $\delta E \cdot w=\hbar / 2$. For the above theoretical calculation to be valid, that is, for the distortion of the reflected wave packet to be negligible, it is required that $\delta E \leq \Delta E$. This results in a restriction on the thickness of the barrier,

$$
a \leq 2 v_{c} w \sin ^{-1} \frac{k_{0}\left|k_{1}-k_{2}\right|}{\left[\left(k_{1}^{2}-k_{0}^{2}\right)\left(k_{2}^{2}-k_{0}^{2}\right)\right]^{1 / 2}} .
$$

Because of the analogy between Schrödinger's equation in quantum mechanics and Helmholtz's equation in electromagnetism [4, 5, 33, the predictions presented here have been observed in a socalled G-band waveguide of width $47.5 \mathrm{~mm}$ by H. Spieker of Braunschweig University in Germany 41, where the asymmetric barrier structure was obtained by reducing the inside width of the waveguide, leading to effective widths of $40.5 \mathrm{~mm}$ and $30.5 \mathrm{~mm}$. The resonance-enhancement of the times is clearly shown, and both the positive and negative resonant peaks of the reflection time is much larger than the resonant peak of the transmission time.

In a word, the reflection and transmission group delays in an asymmetric single quantum barrier are greatly enhanced by the transmission resonance when the energy of incident particles is larger than the height of the barrier. The reflection group delay can be negative as well as positive, depending on the relative height of the potential energies on the two sides of the barrier. The negative resonant reflection group delay corresponds to a transmission probability that is larger than 1. The resonant transmission group delay is of the order of the quasibound state lifetime in the 


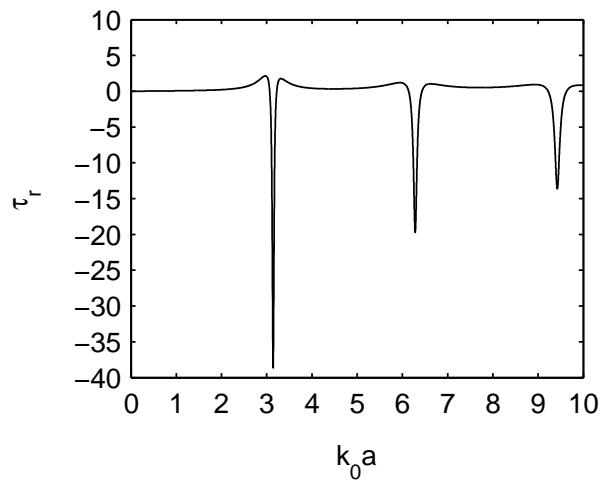

Figure 3: The dependence of the reflection group delay $\tau_{r}$ on the energy $E$ of incident particles, where $V_{1}=0, V_{2}=0.3 V_{0}, E \in\left[V_{0}, 1.15 V_{0}\right], a=10 /\left(0.3 \mu V_{0}\right)^{1 / 2}, E$ is re-scaled to be $k_{0} a$, and $\tau_{r}$ is in units of $\tau_{c}$.

barrier region and is larger than the classical traversal time. The magnitude of resonant reflection group delay is much larger than the lifetime. These phenomena may have potential applications in electronic devices, such as novel quantum-mechanical delay lines. It should be pointed out that the negative reflection group delay does not imply a negative propagation velocity. As a matter of fact, the negative group delay results from the reshaping [17, 42] of the reflected wave packet due to the different phase shifts $\phi_{2}-\phi_{1}$ for its different Fourier components.

\section{Acknowledgments}

The author thanks G. Nimtz for his helpful discussions and suggestions. This work was supported in part by the National Natural Science Foundation of China (Grants 60377025 and 60407007), Shanghai Municipal Education Commission (Grants 01SG46 and 04AC99), Science and Technology Commission of Shanghai Municipal (Grants 03QMH1405 and 04JC14036), and the Shanghai Leading Academic Discipline Program. 


\section{References}

[1] E. H. Hauge and J. A. Støvneng, Rev. Mod. Phys. 61, 917 (1989).

[2] R. Landauer and Th. Martin, Rev. Mod. Phys. 66, 217 (1994).

[3] G. Nimtz and W. Heitmann, Prog. Quantum Electron. 21, 81 (1997).

[4] R. Y. Chiao and A. M. Steinberg, in Progress in Optics, edited by E. Wolf (Elsevier, Amsterdam, 1997), Vol. XXXVII, p. 345.

[5] G. Nimtz, Prog. Quantum Electron. 27, 417 (2003)

[6] E. H. Visscher, J. Lindeman, S. M. Verbrugh, P. Hadley, J. E. Mooij, and W. van der Vlueten, Appl. Phys. Lett. 68, 2014 (1996).

[7] E. R. Brown, J. R. Söderström, C. Parker, L. J. Mahoney, K. M. Molvar, and T. C. McGill, Appl. Phys. Lett. 58, 2291 (1991).

[8] S. Slivken, Z. Huang, A. Evans, and M. Razeghi, Appl. Phys. Lett. 80, 4091 (2002).

[9] E. Luna, M. Hopkinson, J. M. Ulloa, A. Guzmán, and E. Muñoz, Appl. Phys. Lett. 83, 3111 (2003).

[10] M. Büttiker and R. Landauer, Phys. Rev. Lett. 49, 1739 (1982).

[11] M. Büttiker, Phys. Rev. B 27, 6178 (1983).

[12] B. Ricco and M. Ya. Azbel, Phys. Rev. B 29, 1970 (1984).

[13] E. H. Hauge, J. P. Falck, and T. A. Fjeldly, Phys. Rev. B 36, 4203 (1987).

[14] C. R. Leavens and G. C. Aers, Phys. Rev. B 40, 5387 (1989).

[15] A. M. Steinberg and R. Y. Chiao, Phys. Rev. A 49, 3283 (1994).

[16] V. V. Paranjape, Phys. Rev. B 52, 10740 (1995).

[17] Y. Japha and G. Kurizki, Phys. Rev. A 53, 586 (1996).

[18] H. G. Winful, Phys. Rev. E 68, 016615 (2003); Phys. Rev. Lett. 90, 023901 (2003); 91, 260401 (2003).

[19] M. Büttiker and S. Washburn, Nature (London) 422, 271 (2003).

[20] For a review until 1989, see R. Landauer, Nature 341, 567 (1989). 
[21] A. M. Steinberg, P. G. Kwiat, and R. Y. Chiao, Phys. Rev. Lett. 71, 708 (1993).

[22] G. Nimtz, A. Enders, and H. Spieker, J. Phys. I (France) 4, 565(1994).

[23] Ch.Spielmann, R. Szipöcs, A. Stingl, and F. Krausz, Phys. Rev. Lett. 73, 2308 (1994).

[24] A. Ranfagni, D. Mugnai, P. Fabeni, and G. P. Pazzi, Appl. Phys. Lett. 58, 774 (1991).

[25] S. K. Sekatskii and V. S. Letokhov, Phys. Rev. B 64, 233311 (2001).

[26] D. Dragoman and M. Dragoman, J. Appl. Phys. 93, 6133 (2003).

[27] J. C. Martinez and E. Polatdemir, Appl. Phys. Lett. 84, 1320 (2004).

[28] Ph. Balcou and L. Dutriaux, Phys. Rev. Lett. 78, 851(1997).

[29] A. Haibel and G. Nimtz, Ann. Phys. (Leipzig) 10, 707 (2001).

[30] J. C. Martinez and E. Polatdemir, Physica Scripta 68, 108 (2003).

[31] T. E. Hartman, J. Appl. Phys. 33, 3427 (1962).

[32] C.-F. Li and Q. Wang, Phys. Lett. A 275, 287 (2000); X. Chen and C.-F. Li, Phys. Rev. A 68, 052105 (2003).

[33] R.-M. Vetter, A. Haibel, and G. Nimtz, Phys. Rev. E 63, 046701 (2001).

[34] J. G. Muga, I. L. Egusquiza, J. A. Damborenea, and F. Delgado, Phys. Rev. A 66, 042115 (2002).

[35] Ph. Grossel, F. Depasse, and J.-M. Vigoureux, J. Phys. A: Math. Gen. 35, 9787 (2002).

[36] M. Büttiker, in Electronic Properties of Multilayers and low Dimensional Semiconductors, edited by J. M. Chamberlain, L. Eaves, and J. C. Portal (Plenum Press, New York, 1990), p. 297.

[37] S. Longhi, Phys. Rev. E 64, 037601 (2001).

[38] H. Luo, N. Dai, F. C. Zhang, N. Samarth, M. Dobrowolska, J. K. Furdyna, C. Parks, and A. K. Ramdas, Phys. Rev. Lett. 70, 1307 (1993).

[39] E. P. Wigner, Phys. Rev. 98, 145 (1955).

[40] L. D. Landau and E. M. Lifshitz, Quantum Mechanics, Non-relativistic Theory, 3rd edition (Pergamon, Oxford, 1977), p. 77.

[41] H. Spieker, private communication.

[42] A. Dogariu, A. Kuzmich, H. Cao, and L. J. Wang, Optics Express 8, 344 (2001). 